\title{
Physiological Basis of Propagation studies in Commercially Important Difficult to Root Ornamental Shrub Thuja orientais
}

\author{
Aishwarya Kundargi ${ }^{1}$, Sateesh R. Patil ${ }^{1}$, S. G. Gollagi ${ }^{2} *$, \\ B. C. Patil and G. K. Seetharamu
}

${ }^{1}$ Department of Floriculture and Landscape Architecture, Kittur Rani Chanamma College of Horticulture, Arabhavi, University of Horticultural Sciences Bagalkot, India

${ }^{2}$ Department of Crop Physiology, College of Horticulture Bagalkot, India

*Corresponding author

Keywords

Root Ornamental Shrub,

Thuja orientais

Article Info

Accepted:

15 December 2019

Available Online:

20 January 2020

\section{A B S T R A C T}

Among different types of cutting, tip cuttings showed early sprouting, highest number of sprouts per cutting per cutting and length of longest sprout. Subsequently, highest number of primary roots per cutting, secondary roots and length of longest root were found with same type of cutting. Among different levels of IBA, IBA at 4000 ppm resulted in early sprouting, maximum number of sprouts per cutting per cutting and length of longest sprout. Subsequently, highest number of primary roots per cutting, secondary roots and length of longest root were found with same treatment. Among two different media used soil + sand + cocopeat + VAM media recorded early sprouting, maximum number of sprouts per cutting per cutting and length of longest sprout. Subsequently, highest number of primary roots per cutting, secondary roots and length of longest root were found with same treatment.

\section{Introduction}

Vegetative propagation understands the propagation of plant which is produced with the aid of some parts drawed from vegetative organs of plant, so named cuttings. The specimens obtained by that way conserve the biomorphological characters and the heredity peculiarities of the species. For that reason, vegetative propagation found a large implementation in modern horticulture with a view of producing decorative planting material (Leakey et al., 1990).

Thuja orientalis, commonly known as white cedar or arborvitae or northern white cedar, a member of Cupressaceae family, is native to North America. In ornamental arrangements it 
is usually found as shrub reaching up to $12-20$ $\mathrm{m}$ height. The trees are characteristically coniferous and monoecious, have a pyramidshaped with monopodial branchings to the trunk, which is erect with a reddish-brown cortex. The branches are flattened, short, horizontals and covered with small rigid leaves, which overlap one another. The leaves are oval-shaped, persistent, green in color with scales crossing from opposite sides. $T$. orientalis is widely used as an ornamental tree, particularly for screens and hedges, in gardens, parks and cemeteries. Thuja plants are planted in parks and gardens, due to its decorative aspect and can be easily modeled into different and desired shapes. Occasionally Thuja shrubs are propagated through seeds, but can also be easily propagated using vegetative methods, the most common being heel cuttings (Posta and Hernea, 2008).

\section{Materials and Methods}

\section{Preparation of rooting hormone solution}

A 4000 ppm IBA solution was prepared by dissolving one gram of IBA in little quantity of $0.1 \mathrm{~N} \mathrm{NaOH}$ solution, simultaneously 250 $\mathrm{g}$ of talc powder paste is made by adding 250 $\mathrm{ml}$ of water and IBA solution and later the paste was allowed to dry. After drying it was powdered and sieved. Similarly 1000, 2000 and $3000 \mathrm{ppm}$ powder was prepared by taking $0.25 \mathrm{~g}, 0.5 \mathrm{~g}$ and $0.75 \mathrm{~g}$ of IBA respectively.

\section{Treating cuttings and planting}

The method adopted for treating of cuttings with rooting hormone powder, in which, the basal end of the prepared cuttings were kept standing in powder of rooting hormone to a depth of 2.5-3.0 cm. Twenty five cuttings for each treatment were treated with the rooting hormone and repeated thrice in Completely Randomized Design with Factorial concept.
The treated cuttings were planted in portrays containing growing media soil + sand + cocopeat and soil + sand + cocopeat + VAM (the VAM culture used in the study is Acualospors laevis) and placed under mist house as per the treatment. Watering was given through misters for two minutes at an interval of 30 minutes.

\section{Results and Discussion}

\section{Effect of type of cutting}

\section{Shoot characters}

Type of cuttings showed prominent influence on various shoot characters. Minimum days for sprouting (13.87 days) and number of sprouts per cutting (11.34) was observed in tip cuttings. This might be due to active leaf is necessary to provide energy for the growth of stem cuttings, as there is little reserves in the stem Reuveni and Raviv (1980). Similar reports on shoot characters were also noted by Soga et al. (2018) in Thuja orientalis and Chowdhuri et al. (2017) in Cape jasmine.

\section{Root characters}

Number of primary roots per cutting (4.06) and rooting percentage $(51.00 \%)$ was significantly influenced by type of cuttings. As rooting is stimulated by high levels of available carbohydrates provided by the leaves. The findings were in accordance with Griffin et al. (1998) in Thuja orientalis and Chowdhuri et al. (2017) in Cape jasmine.

\section{Effect of rooting hormone}

\section{Shoot characters}

The result observed that minimum days taken to sprouting (13.47 days) and highest number of sprouts per cutting (10.53) was significantly noted with 4000 ppm IBA 
application. This might be due to early rooting and favorable environment showed different response based on concentration of plant growth regulators, viz. IBA 4000 ppm was found best optimum concentration for root initiation. Similar reports on sprouting of roots were also noted by Deshmukh and Barad (2002) in Bougainvillea and (Singh et al., 2009) in Guggal.

\section{Root characters}

Among the different IBA treatment, 4000 ppm IBA was the most effective as it enhanced the maximum number of primary (4.87) and rooting $(51.33 \%)$. The better rooting and root growth with auxin might be ascribed due to greater metabolic activity and maximum utilization of sugar and starch after hydrolysis from stem has been experimentally substantiated by various researcher like Hirapara et al. (2007) in Jasmine and Torkashv and Shadparvar (2011) in Hibiscus.

\section{Effect of growing media}

\section{Shoot characters}

Among two growing media, minimum days for sprouting (15.03 days) and highest number of sprouts per cutting (7.71) was recorded in soil + sand + cocopeat + VAM. This might be due to Arbuscular mycorrhizal fungi are well known to improve the nutritional status and thereby aid in increased growth of plants. Similar findings were noticed by Bhatti et al. (2013) in Carnation.

\section{Root characters}

Soil + sand + cocopeat + VAM media recorded significantly highest number of primary roots per cutting (3.64) and rooting $(38.57 \%)$ which was primarily due to correlation of root parameters with mycorrhizal inoculation amount of root is probably related to suitable ventilation of soil that is the result of hypha network of mycorrhizal fungi that connects particles of soil and as result the rootspreads into deep soil and produce more of roots. Similar reports were noted by Davies (1987) in Rose and Kerur and Lakshman (2009) in Carnation.

Interaction effect of type of cuttings and rooting hormone

\section{Shoot characters}

Application of $4000 \mathrm{ppm}$ of IBA to tip cuttings resulted in minimum days taken for sprout initiation (11.60 days) and highest number of sprouts per cutting (15.56). This may be due to greater stability and low mobility of IBA which induces all growth responses. These results came in conformity with the findings of Parmar et al. (2010) in Bougainvillea and Shadparvar et al. (2011) in Hibiscus.

\section{Root characters}

Highest number of primary roots per cutting (5.70) and rooting per cent $(66.00 \%)$ was observed in tip cuttings with application of $4000 \mathrm{ppm}$ of IBA. The initiation and development of roots on stem cuttings is controlled by complex ecophysiological and biochemical processes, as well as anatomical factors and exogenous application of IBA helps in enhancing these factors as well mobilize sucrose or its derivative to the site of root formation. The results were in agreement with Shirol et al. (1992) in Poinsettia.

\section{Interaction effect of type of cuttings and growing media}

\section{Shoot characters}

Significantly minimum days taken for sprout intiation (13.45 days) was noticed in tip 
cuttings planted in soil + sand + cocopeat + VAM media which was due to mycorrhizal fungi can enhance qualification and quantification characteristics in cuttings. This was in accordance with the reports of Bhatti et al. (2012) in Carnation and Bidarnamani and Mohkami (2014) in Rosemary.

\section{Root characters}

From the data it is evident that highest number of primary roots per cutting (4.16) and rooting (52.80) were observed in tip cuttings planted in soil + sand + cocopeat + VAM media which was due to mycorrhizal fungi can enhance qualification and quantification characteristics in cuttings. This was in conformity with the results of Bhatti et al. (2012) in Carnation and (Bidarnamani and Mohkami, 2014) in Rosemary.

\section{Interaction effect of rooting hormone and growing media}

\section{Shoot characters}

Minimum days taken for sprout initiation (13.00 days) and highest number of sprouts per cutting (10.73) was found in cuttings planted in soil + sand + cocopeat + VAM treated with $4000 \mathrm{ppm}$ of IBA this might be due to mycorrhizal fungi has symbiotic association and improves the growth of plants through enhanced uptake of macro and micronutrient as well as improves plant resistance against biotic and abiotic stress. Similar findings were reported by Amri (2015) in Dalbergia melanoxylon and Adams et al. (2018) in Thuja orientalis.

\section{Root characters}

Highest number of primary roots per cutting (5.02) and rooting $(53.33 \%)$ was seen soil + sand + cocopeat + VAM treated with 4000 ppm of IBA this might be due to IBA assist the colonization of a host (mycorrhiza fungi) by increasing the number of lateral roots as preferred colonization sites for the fungi during early growth development. Similar results were also obtained by Amri (2015) in Dalbergia melanoxylon and Adams et al. (2018) in Thuja orientalis.

\section{Interaction effect of type of cutting, rooting} hormone and growing media

\section{Shoot characters}

Minimum days taken for sprout initiation (11.13 days) and number of sprouts per cutting (15.93) was found in tip cuttings planted in soil + sand + cocopeat + VAM treated with $4000 \mathrm{ppm}$ of IBA this is due to interaction effect enhance the utilization of carbohydrates at the base of cuttings through creation of sink and better utilization of photosynthesis and enhance uptake of nutrients leading to early growth. The results were in accordance with Parmar et al. (2010) in Bougainvillea; Abidin and Metali (2015) in Martelli Shrub and Adams et al. (2018) in Thuja orientalis.

\section{Root characters}

Highest number of primary roots per cutting (5.80) and rooting (68.00\%) was found in tip cuttings planted in soil + sand + cocopeat + VAM treated with $4000 \mathrm{ppm}$ of IBA this is due to mycorrhizal fungi influence on rooting ability of auxin treated stem cuttings. The results were in conformity with Bidarnamani and Mohkami (2014) in Rosemary; and Bhattacharjee and Balakrishana (1992) in Hamelia patens.

Based on the results of the present investigation, it can be concluded that tip cutting is most effective for rooting of cuttings whereas, 4000 ppm IBA is the most effective for rooting of cuttings. Soil + sand + cocopeat + VAM media is more beneficial for rooting of cuttings of Thuja orientalis. 
Table.1 Days taken for sprout initiation as influenced by type of cuttings and rooting hormone and their interaction effect in Thuja orientalis

\begin{tabular}{|c|c|c|c|c|c|c|}
\hline \multirow[t]{2}{*}{ Type of cutting } & $\mathbf{T}_{\mathbf{0}}$ & \multicolumn{3}{|c|}{ Rooting hormone (ppm) } & $\mathbf{T}_{4}$ & \multirow[t]{2}{*}{ Mean } \\
\hline & (control) & $\begin{array}{c}T_{1} \\
(\mathbf{1 0 0 0})\end{array}$ & $\begin{array}{c}\mathbf{T}_{2} \\
(\mathbf{2 0 0 0})\end{array}$ & $\begin{array}{c}\mathbf{T}_{3} \\
(\mathbf{3 0 0 0})\end{array}$ & $(4000)$ & \\
\hline $\mathrm{C}_{1}-\mathrm{Tip}$ & 15.73 & 15.20 & 14.03 & 12.80 & 11.60 & 13.87 \\
\hline $\mathrm{C}_{2}$-Semi hardwood & 16.86 & 16.50 & 14.76 & 14.50 & 13.66 & 15.26 \\
\hline $\mathrm{C}_{3}$-Hardwood & 19.30 & 18.16 & 16.96 & 16.16 & 15.16 & $\mathbf{1 7 . 1 5}$ \\
\hline Mean & 17.30 & 16.62 & 15.25 & 14.48 & 13.47 & \\
\hline Sources & \multicolumn{3}{|c|}{ S.Em \pm} & \multicolumn{3}{|c|}{ C.D (5\%) } \\
\hline $\mathbf{C}$ & \multicolumn{3}{|c|}{0.02} & \multicolumn{3}{|c|}{0.06} \\
\hline $\mathbf{T}$ & \multicolumn{3}{|c|}{0.02} & \multicolumn{3}{|c|}{0.07} \\
\hline $\mathbf{C} \times \mathbf{T}$ & \multicolumn{3}{|c|}{0.04} & \multicolumn{3}{|c|}{0.13} \\
\hline
\end{tabular}

Table.2 Days taken for sprout initiation as influenced by type of cuttings and growing media and their interaction effect in Thuja orientalis

\begin{tabular}{|c|c|c|c|}
\hline \multirow[b]{2}{*}{ Type of cutting } & \multicolumn{3}{|c|}{ Growing media } \\
\hline & $\begin{array}{c}\mathrm{M}_{1} \\
+ \text { Sand +Cocopeat) }\end{array}$ & $($ Soil + Sand + Cocopeat + VAM) & Mean \\
\hline $\mathrm{C}_{1}-\mathrm{Tip}$ & 14.29 & 13.45 & 13.87 \\
\hline $\mathrm{C}_{2}$-Semi hardwood & 15.69 & 14.82 & 15.26 \\
\hline $\mathrm{C}_{3}-$ Hardwood & 17.49 & 16.81 & 17.15 \\
\hline Mean & 15.82 & 15.03 & \\
\hline Sources & S.Em \pm & C.D (5\%) & \\
\hline C & 0.02 & 0.06 & \\
\hline $\mathbf{M}$ & 0.02 & 0.06 & \\
\hline $\mathrm{C} \times \mathrm{M}$ & 0.03 & 0.09 & \\
\hline
\end{tabular}

Table.3 Days taken for sprout initiation as influenced by rooting hormone and growing media and their interaction effect in Thuja orientalis

\begin{tabular}{|c|c|c|c|}
\hline \multirow{3}{*}{$\begin{array}{l}\text { Rooting hormone } \\
\text { (ppm) }\end{array}$} & \multicolumn{2}{|c|}{ Growing media } & \multirow{3}{*}{ Mean } \\
\hline & $\mathbf{M}_{1}$ & $\mathbf{M}_{2}$ & \\
\hline & (Soil + Sand Cocopeat) & (Soil + Sand +Cocopeat+VAM) & \\
\hline $\mathrm{T}_{0}$ (control) & 17.64 & 16.95 & 17.30 \\
\hline $\mathrm{T}_{1}(1000)$ & 17.06 & 16.17 & 16.62 \\
\hline $\mathrm{T}_{2}(2000)$ & 15.57 & 14.93 & 15.25 \\
\hline $\mathrm{T}_{3}(3000)$ & 14.88 & 14.08 & 14.48 \\
\hline $\mathrm{T}_{4}(4000)$ & 13.95 & 13.00 & 13.47 \\
\hline Mean & 15.82 & 15.03 & \\
\hline Sources & S.Em \pm & \multicolumn{2}{|l|}{ C.D $(5 \%)$} \\
\hline $\mathbf{T}$ & 0.02 & \multicolumn{2}{|l|}{0.07} \\
\hline $\mathbf{M}$ & \multirow{2}{*}{0.02} & \multicolumn{2}{|l|}{0.06} \\
\hline $\mathrm{T} \times \mathrm{M}$ & & \multicolumn{2}{|l|}{0.12} \\
\hline
\end{tabular}


Table.4 Interaction effect of type of cutting, rooting hormone and growing media on days taken for sprout initiation in Thuja orientalis

\begin{tabular}{|c|c|c|c|c|c|c|c|c|c|c|}
\hline & \multicolumn{5}{|c|}{$\begin{array}{c}\mathrm{M}_{1} \\
\text { (Soil + Sand + Cocopeat })\end{array}$} & \multicolumn{5}{|c|}{$\begin{array}{c}\mathrm{M}_{2} \\
(\text { Soil }+ \text { Sand + Cocopeat + VAM })\end{array}$} \\
\hline & $\begin{array}{c}\mathrm{T}_{0} \\
\text { (control) }\end{array}$ & $\begin{array}{c}T_{1} \\
(1000 \text { ppm) }\end{array}$ & $\begin{array}{c}\mathrm{T}_{2} \\
(2000 \mathrm{ppm})\end{array}$ & $\begin{array}{c}T_{3} \\
(3000 \mathrm{ppm}) \\
\end{array}$ & $\begin{array}{c}\mathrm{T}_{4} \\
(\mathbf{4 0 0 0} \mathrm{ppm}) \\
\end{array}$ & $\begin{array}{c}\mathrm{T}_{0} \\
\text { (control) }\end{array}$ & $\begin{array}{c}\mathrm{T}_{1} \\
(\mathbf{1 0 0 0} \mathrm{ppm}) \\
\end{array}$ & \begin{tabular}{|c|}
$\mathbf{T}_{2}$ \\
$(2000$ ppm $)$ \\
\end{tabular} & $\begin{array}{c}\mathrm{T}_{3} \\
(3000 \mathrm{ppm}) \\
\end{array}$ & $\begin{array}{c}\mathrm{T}_{4} \\
(4000 \mathrm{ppm})\end{array}$ \\
\hline $\mathrm{C}_{1}$ & 16.13 & 15.73 & 14.26 & 13.26 & 12.06 & 15.33 & 14.66 & 13.80 & 12.33 & 11.13 \\
\hline $\mathrm{C}_{2}$ & 17.13 & 16.86 & 15.26 & 14.93 & 14.26 & 16.60 & 16.13 & 14.26 & 14.06 & 13.06 \\
\hline $\mathrm{C}_{3}$ & 19.66 & 18.60 & 17.20 & 16.46 & 15.53 & 18.93 & 17.73 & 16.73 & 15.86 & 14.80 \\
\hline Sources & \multicolumn{5}{|c|}{ S.Em \pm} & \multicolumn{5}{|c|}{ C.D (5\%) } \\
\hline $\mathbf{C} \times \mathbf{T} \times \mathbf{M}$ & \multicolumn{5}{|c|}{0.06} & \multicolumn{5}{|c|}{0.18} \\
\hline
\end{tabular}

$\mathbf{C}_{1}$ : Tip cutting; $\mathbf{C}_{2}$ : Semi hardwood cutting; $\mathbf{C}_{3}$ : Hardwood cutting 
Int.J.Curr.Microbiol.App.Sci (2020) 9(1): 2180-2194

Table.5 Number of sprouts as influenced by type of cutting and rooting hormone and their interaction effect in Thuja orientalis

\begin{tabular}{|c|c|c|c|c|c|c|c|c|c|c|c|c|c|c|c|c|c|c|}
\hline & \multicolumn{15}{|c|}{ Rooting hormone (ppm) } & \multicolumn{3}{|c|}{ Mean } \\
\hline \multirow{3}{*}{$\begin{array}{l}\text { Type of } \\
\text { cutting }\end{array}$} & \multicolumn{3}{|c|}{$T_{0}$ (control) } & \multicolumn{3}{|c|}{$T_{1}(1000)$} & \multicolumn{3}{|c|}{$T_{2}(2000)$} & \multicolumn{3}{|c|}{$T_{3}(3000)$} & \multicolumn{3}{|c|}{$T_{4}(4000)$} & & & \\
\hline & 60 & 90 & 120 & 60 & 90 & 120 & 60 & 90 & 120 & 60 & 90 & 120 & 60 & 90 & 120 & 60 & 90 & 120 \\
\hline & $\overline{\text { DAP }}$ & $\overline{\mathbf{D A P}}$ & $\overline{\text { DAP }}$ & $\overline{\text { DAP }}$ & \begin{tabular}{|l|} 
DAP \\
\end{tabular} & DAP & $\overline{\text { DAP }}$ & \begin{tabular}{|l|} 
DAP \\
\end{tabular} & $\overline{\mathbf{D A P}}$ & $\overline{\mathbf{D A P}}$ & \begin{tabular}{|l|} 
DAPP \\
\end{tabular} & DAP & $\overline{\text { DAP }}$ & DAP & \begin{tabular}{|l|} 
DAP \\
\end{tabular} & DAP & $\overline{\mathbf{D A P}}$ & $\overline{\mathbf{D A P}}$ \\
\hline $\mathrm{C}_{1}$ & 0.70 & 1.50 & 2.30 & 1.33 & 2.30 & 3.36 & 2.20 & 3.06 & 4.20 & 3.29 & 4.30 & 5.00 & 3.83 & 4.56 & 5.23 & 2.27 & 3.14 & 4.01 \\
\hline $\mathrm{C}_{2}$ & 0.60 & 1.30 & 1.90 & 1.30 & 2.00 & 2.60 & 2.30 & 3.20 & 4.13 & 2.66 & 3.73 & 4.46 & 3.16 & 4.00 & 4.94 & 2.00 & 2.84 & 3.61 \\
\hline $\mathrm{C}_{3}$ & 0.50 & 1.10 & 1.60 & 1.00 & 1.50 & 2.20 & 1.63 & 2.46 & 3.30 & 2.30 & 3.46 & 4.20 & 2.90 & 3.90 & 4.63 & 1.66 & 2.48 & 3.19 \\
\hline Mean & 0.60 & 1.30 & 1.93 & 1.21 & 1.93 & 2.72 & 2.04 & 2.91 & 3.87 & 2.75 & 3.83 & 4.55 & 3.30 & 4.15 & 4.93 & & & \\
\hline \multirow[t]{2}{*}{ Sources } & & & & & S.Em \pm & & & & & & & & & C.D (5 & & & & \\
\hline & \multicolumn{3}{|c|}{$60 \mathrm{DAP}$} & \multicolumn{3}{|c|}{$90 \mathrm{DAP}$} & \multicolumn{3}{|c|}{$120 \mathrm{DAP}$} & \multicolumn{3}{|c|}{$\frac{1}{60 \mathrm{DAP}}$} & \multicolumn{3}{|c|}{90 DAP } & \multicolumn{3}{|c|}{$120 \mathrm{DAP}$} \\
\hline $\mathbf{C}$ & \multicolumn{3}{|c|}{0.009} & \multicolumn{3}{|c|}{0.009} & \multicolumn{3}{|c|}{0.01} & \multicolumn{3}{|c|}{0.02} & \multicolumn{3}{|c|}{0.03} & \multicolumn{3}{|c|}{0.04} \\
\hline $\mathbf{T}$ & \multirow{2}{*}{\multicolumn{3}{|c|}{$\begin{array}{l}0.01 \\
0.02\end{array}$}} & \multirow{2}{*}{\multicolumn{3}{|c|}{$\begin{array}{l}0.01 \\
0.02\end{array}$}} & \multicolumn{3}{|c|}{0.02} & \multicolumn{3}{|c|}{0.03} & \multicolumn{3}{|c|}{0.03} & \multicolumn{3}{|c|}{0.06} \\
\hline $\mathrm{C} \times \mathrm{T}$ & & & & & & & \multirow{2}{*}{\multicolumn{3}{|c|}{$\begin{array}{r}0.03 \\
\text { utting }\end{array}$}} & \multicolumn{3}{|c|}{0.05} & \multicolumn{3}{|c|}{0.06} & \multicolumn{3}{|c|}{0.10} \\
\hline $\mathbf{C}_{1}:$ Tip cl & \multicolumn{3}{|c|}{$\frac{0.02}{\text { ttings; } \mathbf{C}_{2}: \text { Semi hard }}$} & \multicolumn{3}{|c|}{ od cutting; $\mathbf{C}_{3}$ Hard } & & & & & & & & & & & & \\
\hline
\end{tabular}

Table.6 Number of sprouts as influenced by type of cutting and growing media and their interaction effect in Thuja orientalis

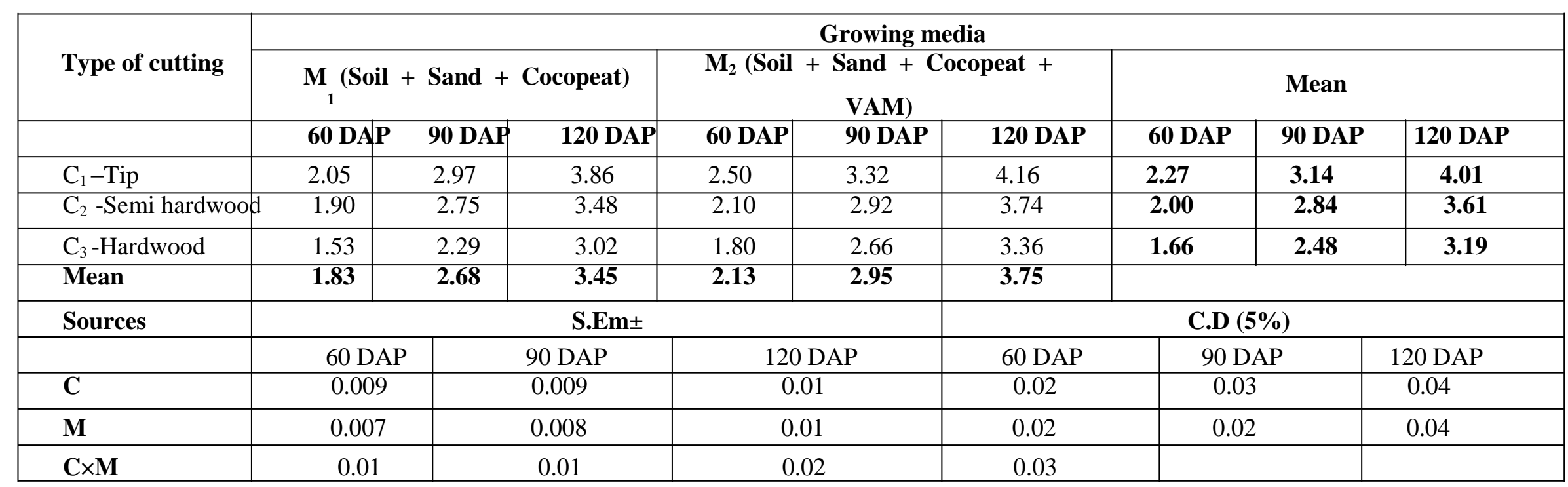


Table.7 Number of sprouts as influenced by rooting hormone and growing media and their interaction effect in Thuja orientalis

\begin{tabular}{|c|c|c|c|c|c|c|c|c|c|}
\hline \multirow{3}{*}{$\begin{array}{c}\text { Rooting } \\
\text { hormone (ppm) } \\
\end{array}$} & \multicolumn{9}{|c|}{ Growing media } \\
\hline & \multicolumn{3}{|c|}{$M_{1}$ (Soil + Sand + Cocopeat) } & \multicolumn{3}{|c|}{$M_{2}($ Soil + Sand + Cocopeat + VAM $)$} & \multicolumn{3}{|c|}{ Mean } \\
\hline & 60 DAP & 90 DAP & 120 DAP & 60 DAP & 90 DAP & \begin{tabular}{|l|} 
120 DAP \\
\end{tabular} & 60 DAP & 90 DAP & 120 DAP \\
\hline $\mathrm{T}_{1}(1000)$ & 1.06 & 1.80 & 2.53 & 1.35 & 2.06 & 2.91 & 1.21 & 1.93 & 2.72 \\
\hline $\mathrm{T}_{2}(2000)$ & 1.88 & 2.73 & 3.68 & 2.20 & 3.08 & 4.00 & 2.04 & 2.91 & 3.87 \\
\hline $\mathrm{T}_{3}(3000)$ & 2.53 & 3.60 & 4.39 & 2.98 & 4.06 & 4.59 & 2.75 & 3.83 & 4.55 \\
\hline Sources & \multicolumn{5}{|c|}{ S.Em \pm} & \multicolumn{4}{|c|}{ C.D (5\%) } \\
\hline & \multicolumn{2}{|c|}{60 DAP } & $90 \mathrm{DAP}$ & \multicolumn{2}{|c|}{120 DAP } & 60 DAP & \multirow{2}{*}{\multicolumn{2}{|c|}{90 DAP }} & 120 DAP \\
\hline $\mathbf{T}$ & \multicolumn{2}{|c|}{0.01} & 0.01 & \multicolumn{2}{|c|}{0.02} & 0.03 & \multirow{2}{*}{\multicolumn{2}{|c|}{$\frac{0.03}{0.02}$}} & 0.06 \\
\hline $\mathbf{M}$ & \multicolumn{2}{|c|}{0.007} & 0.008 & \multicolumn{2}{|c|}{0.01} & 0.02 & & & 0.03 \\
\hline $\bar{T} \times \mathbf{M}$ & \multicolumn{2}{|c|}{0.01} & 0.01 & \multicolumn{2}{|c|}{0.03} & 0.03 & \multicolumn{2}{|l|}{0.03} & 0.09 \\
\hline
\end{tabular}

Table.8 Interaction effect of type of cutting, rooting hormone and growing media on number of sprouts in Thuja orientalis at 60 DAP

\begin{tabular}{|c|c|c|c|c|c|c|c|c|c|c|}
\hline & \multicolumn{5}{|c|}{$M_{1}($ Soil + Sand + Cocopeat $)$} & \multicolumn{5}{|c|}{$M_{2}($ Soil + Sand + Cocopeat + VAM $)$} \\
\hline & $\begin{array}{c}\mathbf{T}_{0} \\
\text { (control) }\end{array}$ & $\begin{array}{c}T_{1} \\
(1000 \text { ppm) }\end{array}$ & $\begin{array}{c}\mathbf{T}_{2} \\
(2000 \text { ppm })\end{array}$ & $\begin{array}{c}\mathbf{T}_{3} \\
(3000 \text { ppm })\end{array}$ & $\begin{array}{c}\mathrm{T}_{4} \\
(\mathbf{4 0 0 0} \\
\mathbf{p p m})\end{array}$ & $\begin{array}{c}\mathbf{T}_{\mathbf{0}} \\
\text { (control) }\end{array}$ & $\begin{array}{c}T_{1} \\
(1000 \mathrm{ppm})\end{array}$ & $\begin{array}{c}\mathbf{T}_{2} \\
(2000 \text { ppm })\end{array}$ & $\begin{array}{c}T_{3} \\
(3000 \text { ppm) }\end{array}$ & $\begin{array}{c}\mathrm{T}_{4} \\
(4000 \mathrm{ppm})\end{array}$ \\
\hline $\mathrm{C}_{1}$ & 0.60 & 1.20 & 2.00 & 2.80 & 3.67 & 0.80 & 1.47 & 2.40 & 3.00 & 4.00 \\
\hline $\mathrm{C}_{2}$ & 0.53 & 1.20 & 2.20 & 2.60 & 3.13 & 0.80 & 1.40 & 2.40 & 2.73 & 3.20 \\
\hline $\mathrm{C}_{3}$ & 0.40 & 0.80 & 1.47 & 2.20 & 2.80 & 0.60 & 1.20 & 1.80 & 2.40 & 3.00 \\
\hline Sources & \multicolumn{5}{|c|}{ S.Em \pm} & \multicolumn{5}{|c|}{ C.D (5\%) } \\
\hline $\mathrm{C} \times \mathbf{T} \times \mathbf{M}$ & \multicolumn{5}{|c|}{0.02} & \multicolumn{5}{|c|}{0.07} \\
\hline
\end{tabular}

$\mathrm{C}_{1}$ : Tip cuttings; $\mathrm{C}_{2}$ : Semi hardwood cutting; $\mathrm{C}_{3}$ Hardwood cutting 
Table.9 Interaction effect of type of cutting, rooting hormone and growing media on number of sprouts in Thuja orientalis at 90 DAP

\begin{tabular}{|c|c|c|c|c|c|c|c|c|c|c|}
\hline & \multicolumn{5}{|c|}{$M_{1}($ Soil + Sand + Cocopeat $)$} & \multicolumn{5}{|c|}{$M_{2}($ Soil + Sand + Cocopeat + VAM $)$} \\
\hline & $\begin{array}{c}\mathbf{T}_{0} \\
\text { (control) }\end{array}$ & $\begin{array}{c}T_{1} \\
(1000 \text { ppm) }\end{array}$ & $\begin{array}{c}T_{2} \\
(2000 \text { ppm) }\end{array}$ & $\begin{array}{c}\mathbf{T}_{3} \\
(3000 \text { ppm) }\end{array}$ & $\begin{array}{c}T_{4} \\
(4000 \\
\text { ppm) }\end{array}$ & $\begin{array}{c}\mathbf{T}_{0} \\
\text { (control) }\end{array}$ & $\begin{array}{c}T_{1} \\
(1000 \\
\text { ppm) }\end{array}$ & $\begin{array}{c}T_{2} \\
(2000 \\
\text { ppm) }\end{array}$ & $\begin{array}{c}T_{3} \\
(3000 \\
\text { ppm) }\end{array}$ & $\begin{array}{c}T_{4} \\
(4000 \\
\text { ppm) }\end{array}$ \\
\hline $\mathrm{C}_{1}$ & 1.40 & 2.20 & 2.87 & 3.80 & 4.33 & 1.60 & 2.40 & 3.27 & 4.00 & 4.80 \\
\hline $\mathrm{C}_{2}$ & 1.20 & 1.80 & 3.00 & 3.60 & 4.20 & 1.40 & 2.20 & 3.40 & 3.87 & 4.40 \\
\hline $\mathrm{C}_{3}$ & 1.00 & 1.40 & 2.33 & 3.40 & 3.80 & 1.20 & 1.60 & 2.60 & 3.53 & 4.00 \\
\hline Sources & \multicolumn{5}{|c|}{ S.Em \pm} & \multicolumn{5}{|c|}{ C.D (5\%) } \\
\hline $\mathrm{C} \times \mathrm{T} \times \mathrm{M}$ & \multicolumn{5}{|c|}{0.07} & \multicolumn{5}{|c|}{0.21} \\
\hline
\end{tabular}

Table.10 Interaction effect of type of cutting, rooting hormone and growing media on number of sprouts in Thuja orientalis at 120 DAP

\begin{tabular}{|c|c|c|c|c|c|c|c|c|c|c|}
\hline & \multicolumn{5}{|c|}{$M_{1}($ Soil + Sand + Cocopeat $)$} & \multicolumn{5}{|c|}{$\mathrm{M}_{2}($ Soil + Sand + Cocopeat + VAM $)$} \\
\hline & $\begin{array}{c}\mathbf{T}_{\mathbf{0}} \\
\text { (control) }\end{array}$ & $\begin{array}{c}\mathrm{T}_{1} \\
(1000 \mathrm{ppm})\end{array}$ & $\begin{array}{c}T_{2} \\
(2000 \text { ppm) }\end{array}$ & $\begin{array}{c}\mathbf{T}_{3} \\
(\mathbf{3 0 0 0} \\
\text { ppm) }\end{array}$ & $\begin{array}{c}T_{4} \\
(4000 \mathrm{ppm})\end{array}$ & $\begin{array}{c}\mathbf{T}_{\mathbf{0}} \\
\text { (control) }\end{array}$ & $\begin{array}{c}T_{1} \\
(1000 \\
\text { ppm })\end{array}$ & $\begin{array}{c}\mathbf{T}_{2} \\
(2000 \\
\text { ppm) }\end{array}$ & $\begin{array}{c}\mathbf{T}_{3} \\
(\mathbf{3 0 0 0} \\
\mathbf{p p m})\end{array}$ & $\begin{array}{c}T_{4} \\
(4000 \\
\text { ppm })\end{array}$ \\
\hline $\mathrm{C}_{1}$ & 3.53 & 4.07 & 4.53 & 5.23 & 6.33 & 3.80 & 4.27 & 4.87 & 5.73 & 6.93 \\
\hline $\mathrm{C}_{2}$ & 2.20 & 3.20 & 4.27 & 5.13 & 5.80 & 2.60 & 3.73 & 4.73 & 5.47 & 6.00 \\
\hline $\mathrm{C}_{3}$ & 3.33 & 4.00 & 4.20 & 5.14 & 5.93 & 3.73 & 4.20 & 4.73 & 5.60 & 6.20 \\
\hline Sources & \multicolumn{5}{|c|}{ S.Em \pm} & \multicolumn{5}{|c|}{ C.D (5\%) } \\
\hline $\mathrm{C} \times \mathrm{T} \times \mathrm{M}$ & \multicolumn{5}{|c|}{0.03} & \multicolumn{5}{|c|}{0.09} \\
\hline
\end{tabular}

C1 : Tip cuttings; $\mathbf{C} 2$ : Semi hardwood cutting; $\mathbf{C 3}$ Hardwood cutting 
Table.11 Number of primary roots as influenced by type of cutting and rooting hormone and their interaction effect in Thuja orientalis

\begin{tabular}{|c|c|c|c|c|c|c|}
\hline \multirow[b]{2}{*}{ Type of cutting } & \multicolumn{5}{|c|}{ Rooting hormone (ppm) } & \multirow[b]{2}{*}{ Mean } \\
\hline & $\begin{array}{c}\mathbf{T}_{\mathbf{0}} \\
\text { (control) }\end{array}$ & $\begin{array}{c}T_{1} \\
(1000)\end{array}$ & $\begin{array}{c}\mathbf{T}_{2} \\
(2000)\end{array}$ & $\begin{array}{c}\mathbf{T}_{3} \\
(\mathbf{3 0 0 0})\end{array}$ & $\begin{array}{c}T_{4} \\
(4000)\end{array}$ & \\
\hline $\mathrm{C}_{1}-\mathrm{Tip}$ & 2.30 & 3.10 & 4.10 & 5.10 & 5.70 & 4.06 \\
\hline $\mathrm{C}_{2}$-Semi hardwood & 2.06 & 2.90 & 3.53 & 3.93 & 4.56 & 3.40 \\
\hline $\mathrm{C}_{3}$-Hardwood & 1.50 & 2.30 & 3.53 & 3.90 & 4.36 & 3.12 \\
\hline Mean & 1.95 & 2.76 & 3.72 & 4.31 & 4.87 & \\
\hline Sources & \multicolumn{3}{|c|}{ S.Em \pm} & \multicolumn{3}{|c|}{ C.D $(5 \%)$} \\
\hline $\mathbf{C}$ & \multicolumn{3}{|c|}{0.01} & \multicolumn{3}{|c|}{0.03} \\
\hline $\mathbf{T}$ & \multicolumn{3}{|c|}{0.01} & \multicolumn{3}{|c|}{0.03} \\
\hline $\mathrm{C} \times \mathrm{T}$ & \multicolumn{3}{|c|}{0.02} & \multicolumn{3}{|c|}{0.06} \\
\hline
\end{tabular}

Table.12 Number of primary roots as influenced by type of cutting and growing media and their interaction effect in Thuja orientalis

Growing media

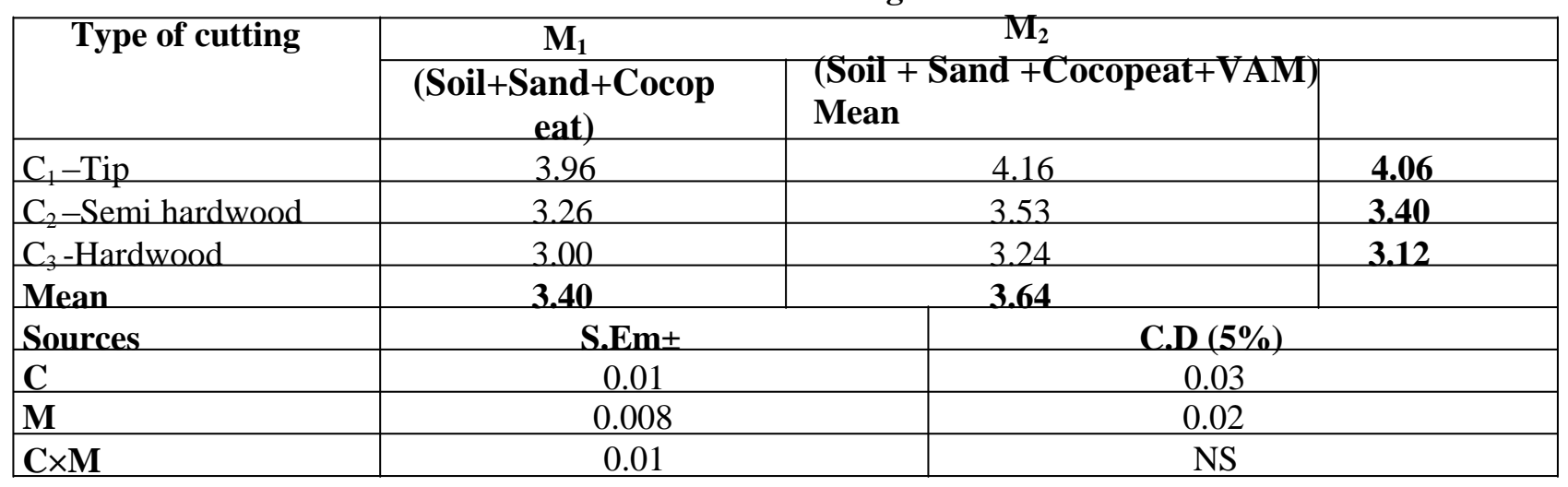

Table.13 Number of primary roots as influenced by rooting hormone and growing media and their interaction effect in Thuja orientalis

Growing media

\begin{tabular}{|c|c|c|c|}
\hline \multirow{3}{*}{$\begin{array}{l}\text { Rooting hormone } \\
\text { (ppm) }\end{array}$} & \multirow{2}{*}{\multicolumn{2}{|c|}{ Growing meala }} & \multirow{3}{*}{ Mean } \\
\hline & & & \\
\hline & (Soil+Sand+Cocopeat) & $($ Soil + Sand +Cocopeat+VAM) & \\
\hline $\mathrm{T}_{0}$ (control) & 1.84 & 2.06 & 1.95 \\
\hline $\mathrm{T}_{1}(1000)$ & 2.66 & 2.86 & 2.76 \\
\hline $\mathrm{T}_{2}(2000)$ & 3.60 & 3.84 & 3.72 \\
\hline $\mathrm{T}_{3}(3000)$ & 4.22 & 4.40 & 4.31 \\
\hline $\mathrm{T}_{4}(4000)$ & 4.71 & 5.02 & 4.87 \\
\hline Mean & 3.40 & 3.64 & \\
\hline Sources & S.Em \pm & \multicolumn{2}{|c|}{ C.D (5\%) } \\
\hline $\mathbf{T}$ & 0.01 & \multicolumn{2}{|l|}{0.03} \\
\hline $\mathbf{M}$ & \multirow{2}{*}{$\begin{array}{c}0.008 \\
0.02\end{array}$} & \multicolumn{2}{|c|}{0.02} \\
\hline T×M & & 0.05 & \\
\hline
\end{tabular}


Table.14 Interaction effect of type of cutting, rooting hormone and growing media on number of primary roots in Thuja orientalis

\begin{tabular}{|c|c|c|c|c|c|c|c|c|c|c|}
\hline & \multicolumn{5}{|c|}{ (Soil + Sand + Cocopeat $)$} & \multicolumn{5}{|c|}{$\begin{array}{c}M_{2} \\
(\text { Soil }+ \text { Sand }+ \text { Cocopeat }+ \text { VAM }) \\
\end{array}$} \\
\hline & $\begin{array}{c}\mathbf{T}_{0} \\
\text { (control) }\end{array}$ & $\begin{array}{c}T_{1} \\
) 0111 \text { ppp }(\end{array}$ & $\begin{array}{c}\mathrm{T}_{2} \\
(2000 \mathrm{ppm})\end{array}$ & $\begin{array}{c}\mathbf{T}_{3} \\
(3000 \mathrm{ppm})\end{array}$ & $\begin{array}{c}T_{4} \\
(4000 \mathrm{ppm})\end{array}$ & $\begin{array}{c}\mathbf{T}_{0} \\
\text { (control) }\end{array}$ & $\begin{array}{c}\mathrm{T}_{1} \\
(1000 \mathrm{ppm})\end{array}$ & $\begin{array}{c}T_{2} \\
(2000 \mathrm{ppm})\end{array}$ & $\begin{array}{c}\mathbf{T}_{3} \\
(\mathbf{3 0 0 0} \mathbf{p p m})\end{array}$ & $\begin{array}{c}\mathrm{T}_{4} \\
(4000 \mathrm{ppm})\end{array}$ \\
\hline $\mathrm{C}_{1}$ & 2.20 & 3.00 & 4.00 & 5.00 & 5.60 & 2.40 & 3.20 & 4.20 & 5.20 & 5.80 \\
\hline $\mathrm{C}_{2}$ & 1.93 & 2.80 & 3.53 & 3.86 & 4.33 & 2.20 & 3.00 & 3.66 & 4.20 & 4.80 \\
\hline $\mathrm{C}_{3}$ & 1.40 & 2.20 & 3.40 & 3.80 & 4.20 & 1.60 & 2.40 & 3.46 & 4.00 & 4.53 \\
\hline Sources & \multicolumn{5}{|c|}{ S.Em \pm} & \multicolumn{5}{|c|}{ C.D (5\%) } \\
\hline $\mathrm{C} \times \mathrm{T} \times \mathrm{M}$ & \multicolumn{5}{|c|}{0.03} & \multicolumn{5}{|c|}{0.09} \\
\hline
\end{tabular}

C1 : Tip cutting; C2: Semi hardwood cutting; C3: Hardwood cutting 
Table.15 Rooting percentage (\%) as influenced by type of cuttings and rooting hormone and their interaction effect in Thuja orientalis

Rooting hormone

\begin{tabular}{|c|c|c|c|c|c|c|}
\hline \multirow{2}{*}{$\begin{array}{l}\text { Type of } \\
\text { cutting }\end{array}$} & $T_{0}$ & $T_{1}$ & $T_{2}$ & $T_{3}$ & $\mathbf{T}_{4}$ & \multirow[b]{2}{*}{ Mean } \\
\hline & (control) & (1000) & (2000) & (3000) & (4000) & \\
\hline $\mathrm{C}_{1}$-Tip & $\begin{array}{l}30.00 \\
(33.18)\end{array}$ & $\begin{array}{l}46.00 \\
(42.68)\end{array}$ & $\begin{array}{c}54.00 \\
(47.27)\end{array}$ & $\begin{array}{c}59.00 \\
(50.16)\end{array}$ & $\begin{array}{c}66.00 \\
(54.31)\end{array}$ & $\begin{array}{c}51.00 \\
(45.52)\end{array}$ \\
\hline $\begin{array}{l}\mathrm{C}_{2} \text {-Semi } \\
\text { hardwood }\end{array}$ & $\begin{array}{c}16.33 \\
(23.79)\end{array}$ & $\begin{array}{c}24.00 \\
(29.32)\end{array}$ & $\begin{array}{c}30.33 \\
(33.39)\end{array}$ & $\begin{array}{c}38.00 \\
(38.03)\end{array}$ & $\begin{array}{c}46.00 \\
(42.68)\end{array}$ & \begin{tabular}{|l|}
$\mathbf{3 1 . 8 0}$ \\
$\mathbf{( 3 3 . 3 4 )}$
\end{tabular} \\
\hline $\mathrm{C}_{3}$-Hardwood & $\begin{array}{r}15.66 \\
(23.27) \\
\end{array}$ & $\begin{array}{c}22.00 \\
(27.93) \\
\end{array}$ & $\begin{array}{c}29.66 \\
(32.97) \\
\end{array}$ & $\begin{array}{c}34.00 \\
(35.64) \\
\end{array}$ & $\begin{array}{c}42.00 \\
(40.37) \\
\end{array}$ & $\begin{array}{l}28.80 \\
(32.14)\end{array}$ \\
\hline Mean & $20.66(26.75)$ & $30.66(33.31)$ & $38.00(37.88)$ & \multicolumn{3}{|c|}{$43.66(41.28) \quad 51.33(45.79)$} \\
\hline Sources & \multicolumn{3}{|c|}{ S.Em \pm} & \multicolumn{3}{|c|}{ C.D (5\%) } \\
\hline $\mathbf{C}$ & \multicolumn{3}{|c|}{0.08} & \multicolumn{3}{|r|}{0.22} \\
\hline $\mathbf{T}$ & \multicolumn{3}{|c|}{0.09} & \multicolumn{3}{|r|}{0.28} \\
\hline $\mathrm{C} \times \mathrm{T}$ & \multicolumn{3}{|c|}{0.17} & \multicolumn{3}{|r|}{0.48} \\
\hline
\end{tabular}

Table.16 Rooting percentage (\%) as influenced by type of cuttings and growing media and their interaction effect in Thuja orientalis

\begin{tabular}{|c|c|c|c|}
\hline \multirow{2}{*}{$\begin{array}{l}\text { Type of } \\
\text { cutting }\end{array}$} & \multicolumn{3}{|c|}{ Growing media } \\
\hline & \begin{tabular}{c|c}
$M_{1}$ \\
(Soil+Sand+Cocopeat)
\end{tabular} & \multicolumn{2}{|r|}{ I) Mean } \\
\hline$C_{1}-$ Tip & $49.20(44.45)$ & $52.80(46.59)$ & $51.00(45.52)$ \\
\hline $\mathrm{C}_{2}$-Semi hardwood & $30.66(33.36)$ & $32.26(34.27)$ & $31.80(33.34)$ \\
\hline $\mathrm{C}_{3}-$ Hardwood & $26.93(30.92)$ & $29.33(32.40)$ & $28.80(32.14)$ \\
\hline Mean & $35.15(35.93)$ & 38.57 (38. & \\
\hline Sources & S.Em \pm & & \\
\hline C & 0.08 & & \\
\hline M & 0.06 & & \\
\hline $\mathbf{C} \times \mathbf{M}$ & 0.11 & & \\
\hline
\end{tabular}

Table.17 Rooting percentage (\%) as influenced by rooting hormone and growing media and their interaction effect in Thuja orientalis

\begin{tabular}{|c|c|c|c|}
\hline \multirow{2}{*}{$\begin{array}{l}\text { Rooting hormone } \\
\text { (ppm) }\end{array}$} & \multicolumn{2}{|c|}{ Growing media } & \\
\hline & $M_{1}$ (Soil+Sand+Cocopeat & $\mathbf{M}_{2}$ (Soil+Sand+Cocopeat+VAM) Mean & \\
\hline $\mathrm{T}_{0}$ (control) & $18.88(25.46)$ & $22.44(28.03)$ & $20.66(26.75)$ \\
\hline $\mathrm{T}_{1}(1000)$ & $29.33(32.47)$ & $32.00(34.16)$ & $30.66(33.31)$ \\
\hline $\mathrm{T}_{2}(2000)$ & $36.22(36.80)$ & $39.77(38.96)$ & $38.00(37.88)$ \\
\hline $\mathrm{T}_{3}(3000)$ & $42.00(40.29)$ & $45.33(42.27)$ & $43.66(41.28)$ \\
\hline $\mathrm{T}_{4}(4000)$ & $49.33(44.62)$ & $53.33(46.96)$ & $51.33(45.79)$ \\
\hline Mean & \multicolumn{2}{|l|}{$35.15(35.93)$} & \begin{tabular}{l|l} 
& $51.33(45.79)$ \\
$38.57(38.08)$
\end{tabular} \\
\hline Sources & \multicolumn{2}{|l|}{ S.Em \pm} & C.D (5\%) \\
\hline $\mathbf{T}$ & \multirow{2}{*}{\multicolumn{2}{|c|}{0.09}} & 0.28 \\
\hline M & & 0.06 & 0.17 \\
\hline $\mathbf{T} \times \mathbf{M}$ & \multicolumn{2}{|l|}{0.14} & 0.39 \\
\hline
\end{tabular}

Values in parenthesis indicates arc sine transformation) 
Int.J.Curr.Microbiol.App.Sci (2020) 9(1): xx-xx

Table.18 Interaction effect of type of cutting, rooting hormone and growing media on rooting percentage (\%) in Thuja orientalis

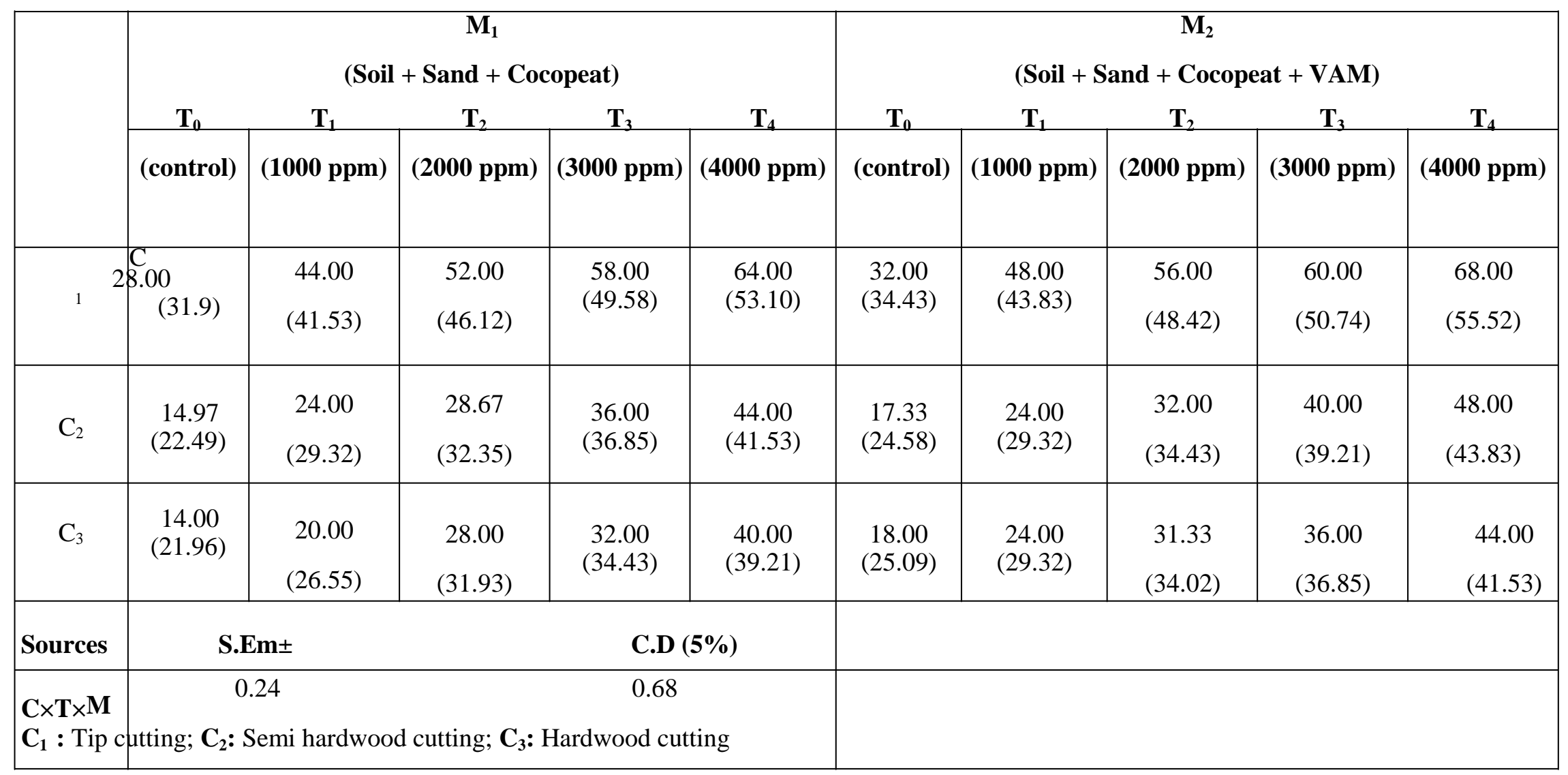

(Values in parenthesis indicates arc sine transformation) 


\section{References}

Abidin N, Metali F. Effects of different types and concentrations of auxins on juvenile stem cuttings for propagation of potential medicinal Dillenia suffruticosa. Martelli Shrub. Res. J Bot. 015; 10:73-87.

Adams, B. A., Elesho, R. O. and Aluko, A. K., 2018, Effect of rooting hormone and growth media on vegetative propagation (Marcotting) of Thuja occidentalis. J. Agric. Sci., 12(2): 33-37.

Amri E. Influence of Arbuscular Mycorrhizal Fungi on rooting ability of auxin treated stem cuttings of Dalbergia melanoxylon (Guill and Perr.). Res. J Bot. 2015; 10:88-97.

Bhattacharjee SK, Balakrishan M. Studies on propagation of Hamelia patens Jacq. from stem cuttings. Prog. Hort. 1992; 24:157-164.

Bhatti SK, Aditya K, Tanuja R, Navroop K. Influence of AM fungi (Glomus mosseae, Acaulospora laevis and Gigaspora sp.) alone and in combination with Trichoderma viride on growth responses and physiological parameters of Dianthus caryophyllus Linn. Adv. Biores. 2013; 4:13-20.

Bidarnamani F, Mohkami Z. Influence of mycorrhizal fungi and cutting type on rooting of cuttings in Rosmarinus officinalis. Indian J Fund. Appl. Sci. 2014; 4(4):2921-2928.

Chowdhuri TK, Sadhukhan RT, Das S. Effect of different growth regulators on propagation of cape jasmine (Tabenaemontana coronaria var. Dwarf) in subtropical zone of West Bengal. Asian J. Hort. 2017; 12(2): 206210.

Davies FT. Effects of VA-mycorrhizal fungi on growth and nutrient uptake of cuttings of Rosa multiflora in two container media with three levels of fertilizer application. J Plant. soil. 1987; 104:31-35.

Deshmukh KK, Barad AV. Effect of IBA and NAA on root initiation of various varieties of bougainvillea species. GAU Res. J. 2001; 27(1\&2):44-46.

Griffin JJ, Blazich FA, Ranney TG. Propagation of thuja cv. 'Green Giant' by stem cuttings: Effect of growth stage, type of cutting and IBA treatment. J. Environ. Horti. 1998; 16(4): 212-214.

Hirapara DV, Parmar BR, Bhayani HN. Effect of IBA and NAA on vegetative propagation of Jasminum arborescens L. cv. Paras through semi hardwood cuttings. Int. J Bio. Sci. Repo., 2007; 5(1):95-99.

Kerur AS, Lakshman HC. Symbiotic response of Dianthus caryophyllus root stock to different mycorrhizal fungi. Int. J Plant. Sci. 2009; 4(1):166-168.

Leakey RR, Mesen JF, Tchoundjeu Z, Dick JM. Low-technology techniques for the vegetative propagation of tropical trees. Commo. Fore. Rev. 1990; 69:247-257.

Parmar BR, Patel VB, Bhalerao PP, Tank RV. Effect of different plant growth regulators on vegetative propagation of Bougainvillea peruviana cv. "Torch Glory" through hardwood cutting. Asian J Hort. 2010; 5(1):222-224.

Posta, D. S., and Hernea, C., 2008, Researches Concerning The production of planting material using vegetative propagation on Thuja plicata D. Don. (Sin. T. gigantea Nutt.). Bulletin UASVM, Horticulture, 65(1): 217-220.

Reuveni O, Raviv M. Importance of leaf retention to rooting of avocado cuttings J Amer. Soc. Hort. Sci. 1980; 106(2):127-130.

Shadparvar V, Mohammadi TA. and Alinejad AH. Effect of IBA and soil mixture on rooting of (Hibiscus rosa-sinensis). Euro. J Exp. Bio. 2011; 1(4): 142-146. 
Shirol AM, Patil AA, Nalawadi VG. Biochemical basis of Euphorbia pulcherima Will. var. Alba. South Ind. Hort. 1992; 40(3):159-156.

Singh J, Kumawat PC, Kumar R, Manmohan JD, Pandey SB, Singh SS. Propagation of guggal (Commiphora wightii Arnott.) bhand through cuttings. Indian $\mathbf{J}$ Agrofor. 2009; 11(2):76-79.
Soga DD, Desma I, Idun A. Rooting response of Thuja occidentalis L. to different soilless media and stem propagation techniques in Ghana. Asian J. Agri. Hort. Res. 2018; 2(2): 1-9.

Torkashvand AM, Shadparvar V. Rooting in Hibiscus rosa sinensis (yellow double hybrid) by indole butyric acid and substrates. IJPAES. 2011; 2(2): 194-197

\section{How to cite this article:}

Aishwarya Kundargi, Sateesh R. Patil, S. G. Gollagi, B. C. Patil and Seetharamu, G. K. 2020. Physiological Basis of Propagation studies in Commercially Important Difficult to Root Ornamental Shrub Thuja orientais. Int.J.Curr.Microbiol.App.Sci. 9(01): 2180-2194.

doi: https://doi.org/10.20546/ijcmas.2020.901.248 